\title{
Real Time Unusual Event Detection in Video Sequences
}

\author{
Aneri Adhvaryu ${ }^{1}$, Kalpesh Jadav ${ }^{2}$ \\ PG Research Scholar, Electronics \& Communication, Parul Institute of Engineering \& Technology, Vadodara, India ${ }^{1}$ \\ Assistant Professor, Electronics \& Communication, Parul Institute of Engineering \& Technology, Vadodara, India ${ }^{2}$
}

\begin{abstract}
Detection of human activities accurately in the video surveillance system is very difficult task. For diverse application areas, while detecting human activities, basically three aspects are taking in concern for human activity recognition system: segmenting object of interest, feature extraction \& representation and human activity detection \& classification. In this paper we have proposed a basic framework for detection suspicious human behaviour as well as tracking of detected human who is doing some unusual activity. The purpose of these methods could lead to better understanding to the suspicious activity of single person as well as multiple persons. The improved design of built environment will provide better surveillance. Such system can be implemented at the public places such as shopping malls, airports, and railway-station or any private premises where security is the prime most concern.
\end{abstract}

Keywords: Video surveillance, Human activity detection \& classification, Unusual event, Skeletonization, Thinning.

\section{INTRODUCTION}

In recent years surveillance systems have used in many applications in public and private premises. Security of the citizens in public places such as Hotels, Markets, Malls, Public Transports is increasingly becoming a crucial task.In recent years, the number of surveillance cameras installed to monitor private and public spaces and areas has increased dramatically.For many visual surveillance applications however, There is a need of such a system that would automatically interpret the entire scene and alert in case of any suspicious situation. Now detection of multiple human activities in real time is very difficult task. Detections of moving objects in video systems is the first step of Information removal in many computer visualization systems for that video cameras are extensively used to examine public and private areas.

Our proposed frame work is to implement a new unusual event detection technique. Human monitoring is tiring, expensive and ineffective. The criteria for the detection of the unusual human behaviour will change according to the scenario. Often times, the object is to detect, recognize or learn events which may be defined as 'suspicious events', 'irregular behaviour', 'uncommon behaviour', 'un-usual activity/event/behaviour', 'abnormal behaviour' etc. The proposed method is based on thinning based skeletonization.Thinning is an image processing operation in which binary valued image regions are reduced to lines that approximate the centre skeletons of the regions [4]. It is required that the lines of the thinned result are connected for each single image region. A common use of thinning is in the pre-processing stage to facilitate higher level analysis and recognition for such applications as Optical Character Recognition, diagram understanding, fingerprint analysis, and feature detection for computer vision systems.

Thinning techniques have been applied in many fields such as automated industrial inspection, pattern recognition, biological shape description and image coding etc. 
B. Yogameena, S.Veeralakshmi [3] proposed a real time video surveillance system which is capable of classifying normal and abnormal action of individuals in crowd. The abnormal action of human such as running, jumping, waving hand, bending, walking and fighting with each other in a crowded environment are considered. In this paper, Relevance Vector Machine(RVM) is used to classify the abnormal actions of individual in crowd based on the results obtained from projection and skeletonization methods.

The authors KamaljeetKaur[4] and Waleed Abu-Ain [5] has given the thinning based method of skeletonization for the binary images. Thinning based skeletonization is another approach for obtaining image skeleton.

\section{III.PROPOSED METHOD}

Proposed method consists of three major classes. i)backgroundmodelling. ii) Skeletonization iii) Unusual event detection. The proposed method is shown in Fig.1. The input video we have taken from Webcam.

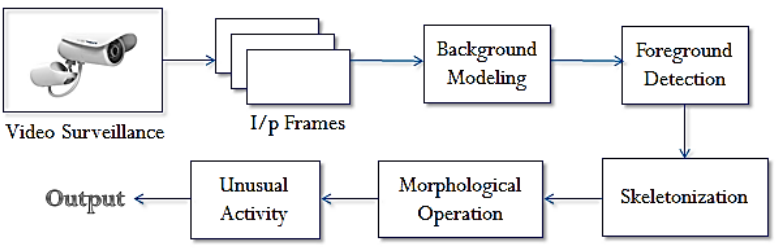

Fig.1 Block Diagram

\section{A) Background Modelling}

Background modelling is a very significant role in a video content analysis system. Background subtraction is the method used to fragment the object in a frame. When static cameras are used, a popular approach is background subtraction, which consists of obtaining a mathematical representation of the static background and comparing it with each new frame from the video sequence. A moving object can be detected easily by identifying parts of the image that do not match with the model. This process is known as background subtraction. It is groundwork for various post-processing modules such as object tracking, recognition, and counting. There are two types of background detection methods: non-adaptive and adaptive. Non-adaptive method depends on definite numbers of video frames and do not sustain a background model in the algorithm. Adaptive methods normally preserve a background model and the parameters of the background model vary over the time. A background model is learned by using Gaussian Mixture Models to represent the time varying back- ground scene. It describes each pixel as mixture of Gaussian and updates the model adaptively according to the input image sequences. The running average approach for the update of the background model Bs is described as follows:

$\mathrm{Bs}=(1-\alpha) \mathrm{Bs}-1+\alpha \mathrm{It}$
Where, $\alpha$ is the pre-determined "learning rate" It is the current pixel intensity value and $\mathrm{Bs}-1$ is the previous running average of the background model. The other parameter of the Gaussian model, the standard deviation $\sigma$, can be updated similarly using the same learning rate $\alpha$ :

$\sigma \mathrm{t}=(1-\alpha) \sigma \mathrm{t}-1+\alpha(\mathrm{It}-\mathrm{Bs})$

Foreground object detection and segmentation from a video sequences is one of the essential task in video surveillance. Foreground pixel are detected by calculating the Euclidean norm,

$H s(u, v)=\left\{\begin{array}{l}1, i f|(u, v)-F(u, v)| S \\ 0, \text { otherwise }\end{array}\right.$

A foreground-background pixel classification method using adaptive thresholding is presented. Thresholding is used to classify a pixel is a foreground or background pixel, and the results are represented using a binary foreground mask $\operatorname{Fg}(\mathrm{x}, \mathrm{y})$,

$\operatorname{Fg}(\mathrm{x}, \mathrm{y})=\left\{\begin{array}{l}1 \text { if It }- \text { Bs }>k \sigma t \\ 0, \text { otherwise }\end{array}\right.$

Where, $\mathrm{k}$ is a constant, It is the pixel intensity of the current frame.

\section{B) Skeletonization}

Skeletonization is transformation of a components of a digital image into a subset of the original components.

Skeletonization algorithms are need to compute a reduced amount of data or to simplify the shape of an object in order to find the features for recognition algorithms and classification. The skeletonization can be computed 1) based on distance transform and specifiedsubsets of the transformed image are a distance skeleton. 2) By thinning approach.

The distance transform is an operator normally only applied to binary images. The result of the transform is a graylevel image that looks similar to the input image, except that the graylevel intensities of points inside foreground regions is changed to show the distance to the closest boundary from each point. One way to think about the distance transform is to first imagine that foreground regions in the input binary image are made of some uniform slow burning inflammable material. Then consider simultaneously starting a fire at all points on the boundary of a foreground region and letting the fire burn its way into the interior. If we then label each point in the interior with the amount of time that the fire took to first reach that point, then we have effectively computed the distance transform of that region.

Binary skeletons can be retrieved by using the MATLAB function bwmorph with the operation set to "thin" and the parameter $\mathrm{N}$, the number of operations to be repeated, set to infinity, which means the operation will be repeatedly 
executed until the image does not change any more. A morphological thinning / is defined as $\mathrm{X} / \mathrm{B}=\mathrm{X} \backslash(\mathrm{X} \times \mathrm{B})$ where $X$ is the hit-or-miss transformation. The bwmorph function uses eight calls to the MATLAB function, eight logical NOTs and eight logical ANDs to perform one round of sequential thinning, and repeat the thinning until the operation no longer generate changes to the binary image.

\section{C) Unusual Event Detection}

In Event detection we have used webcam as a video source to capture sequences of frames and we have applied the background subtraction and skeletonizaton to generate skeleton of an image and then to compare that frame with reference image datasets, Correlation function is used.

Here we are using the MATLAB as a software platform. $\mathrm{r}=\operatorname{corr} 2(\mathrm{~A}, \mathrm{~B})$ returns the correlation coefficient $r$ between $A$ and $B, \quad$ where $A$ and $B$ are matrices or vectors of the same size. $\mathrm{r}$ is a scalar double and it gives the percentage correlation of the two images.

\section{IV.EXPERIMENTAL RESULTS}

Experimental results are shown in Fig.2. Our primary aim is to detect unusual activities in the video sequences, we used input video taken in the Classroom. Background subtraction and Foreground detection process is carried out first as shown in Fig.2.a.

After that applying Skeletonization algorithms on that frame using Thinning based skeletonization process shown in Fig.2.b.

Then we have applied Morphological operation on that frame such as pruning and remove all small components shown in Fig.2.c.and Fig.2.d respectively.

After that Match that Skeleton image frame with the reference frame stored in image Datasets using Correlation Process shown in Fig2.e.

Then if both the Frames correlate with each other, unusual activity detected by setting Red box on that frame shown in Fig2.f.

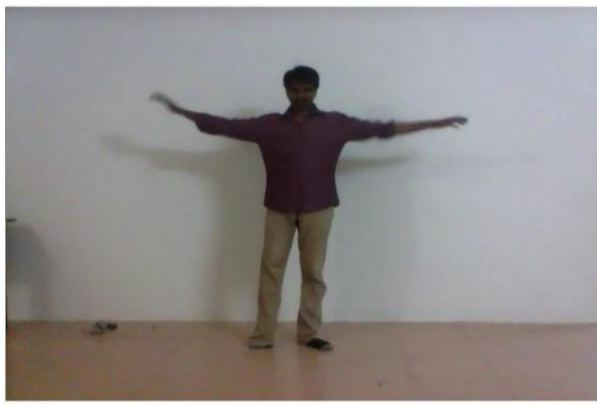

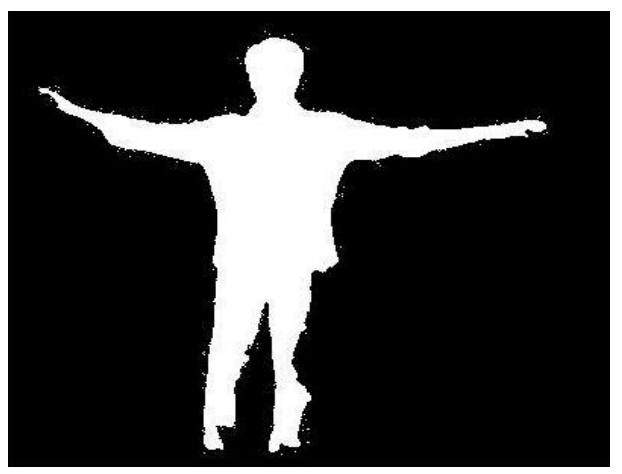

Fig.2.a. Background Subtraction

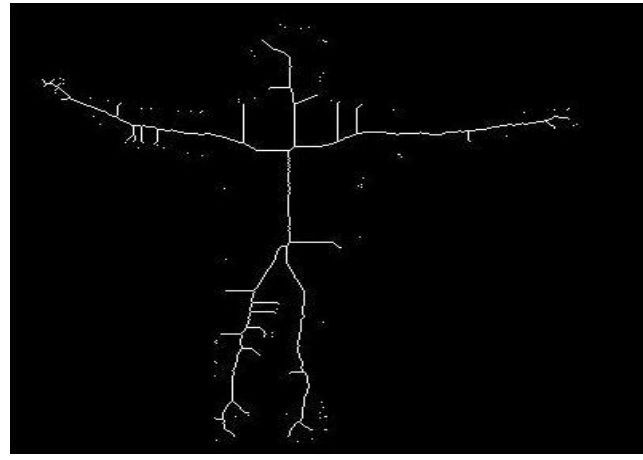

Fig.2.b. Skeletonization (Thinning based)

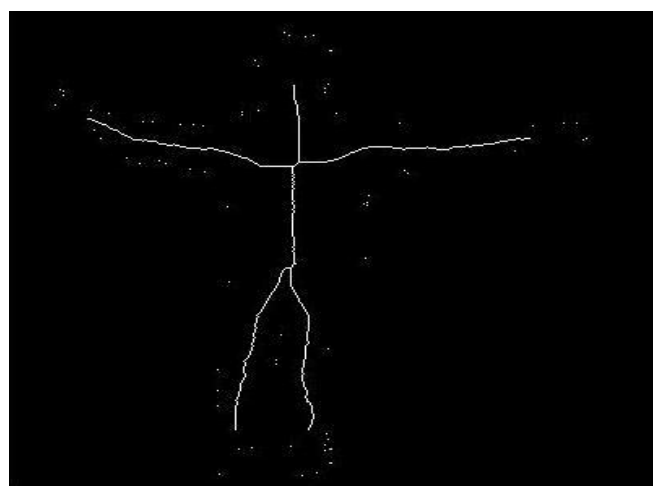

Fig.2.c. Pruning (Morphological Operations)

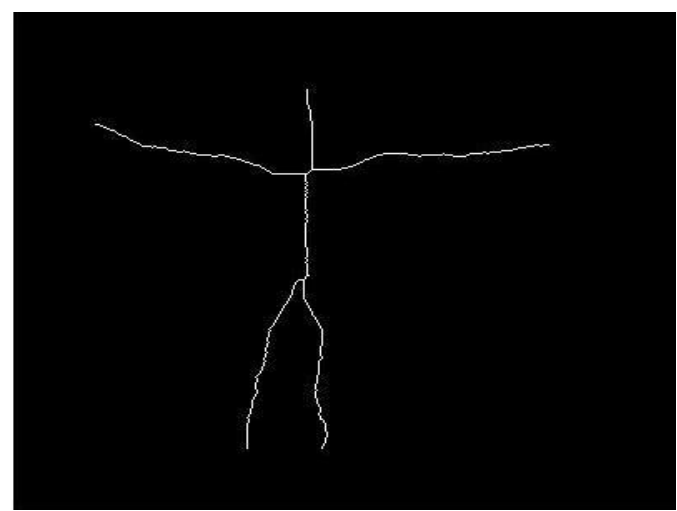

Fig.2.d Removed Small Components

Fig.2. Input Frame 
International Journal of Advanced Research in Computer and Communication Engineering Vol. 4, Issue 3, March 2015

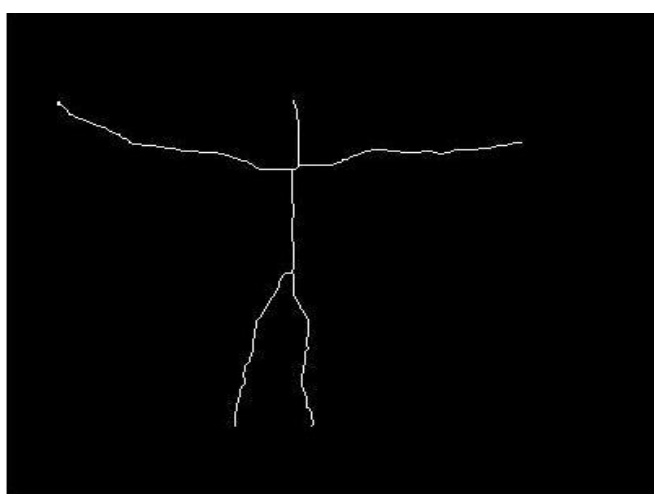

Fig.2.e Reference Image Stored in Datasets

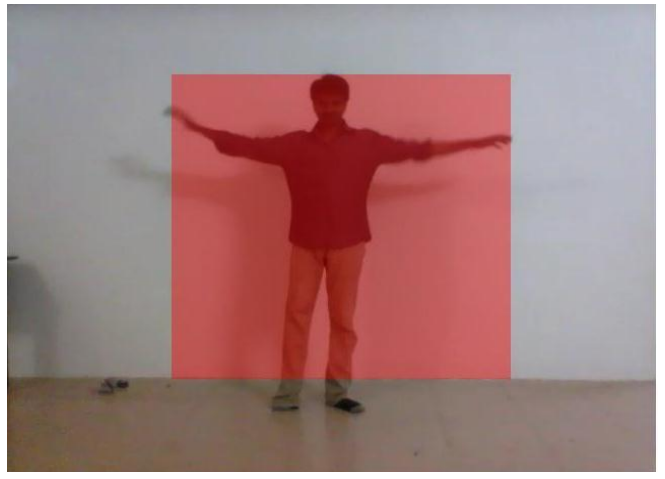

Fig.2.e unusual activity detected

\section{Conclusion}

Detecting Unusual events is very important task in security and monitoring system. We are providing a solution of this problem by using thinning based Image skeletonization method. The proposed method can be applicable in all public and private sectors. The experimental results have demonstrated shows the feasibility and robustness of the proposed system.

\section{REFERENCES}

[1] Ahmed Taha, and Hala H. Zayed, "Skeleton-Based Human Activity Recognition for Video Surveillance," International Journal of Scientific \& Engineering Research, vol. 6, issue 1, January 2015.

[2] Weian Deng, S. Sitharama and Nathan E. Brener, "A Fast Parallel Thinning Algorithm for the Binary Image Skeletonization," International Journal of Hugh Performance Computing Applications, March 2011.

[3] B. Yogameena, "Rvm-Based Human Action Classification in Crowd Through Projection and Star Skeletonization," EURASIP Journal on Image and Video Processing, vol. 2009.

[4] KamaljeetKaur and Mukesh Sharma, "A Method for Binary Image Thinning using Gradient and Watershed Algorithm," International Joiurnal of Advanced Research in Computer Science And Software Engineering, vol.3, issue 1, January 2013.

[5] Waleed Abu-Ain, "Skeletonization Algorithm for Binary Images," The $4^{\text {th }}$ International Conference On Electrical Engineering And Infomatics(ICEEI) 2013. 